\title{
Renormalization Factors of Dimension-six Proton Decay Operators in the Supersymmetric Standard Models
}

Daiki Kobayashi*

Nagoya University

E-mail: kobadth.phys.nagova-u.ac.jp

The renormalization factors of the dimension-six effective operators for proton decay are evaluated at two-loop level in the supersymmetric grand unified theories. For this purpose, we use the previous results in which the quantum corrections to the effective Kähler potential are evaluated at two-loop level. Numerical values for the factors are presented in the case of the minimal supersymmetric SU(5) grand unified model.

KMI International Symposium 2013 on" Quest for the Origin of Particles and the Universe", 11-13 December, 2013

Nagoya University, Japan

\footnotetext{
${ }^{*}$ Speaker.
} 


\section{Introduction}

It is important to make a precise prediction for the decay rate. To that end, we need to determine the effects of the dimension-six operators, which are generated at the GUT scale, on the low-energy physics by using the renormalization group equations (RGEs). Indeed, there have been several literature in which the renormalization factors for the effective operators are evaluated. In Ref. [W], the long-distance QCD corrections are computed at two-loop level. For the short-distance factors, on the other hand, only the one-loop calculation is carried out in Ref. [D] in the SUSY SM.

In this Letter, therefore, we evaluate the renormalization factors of the dimension-six operators at two-loop level in the presence of the supersymmetry. In the calculation, we use the results for the two-loop corrections to the effective Kähler potential given in Ref. [B] Since in the SUSY GUTs, the most of the intermediate energy scales are supersymmetric, the short-distance renormalization factors are well approximated by those evaluated in purely SUSY theory. Thus, combined with the long-distance effects given in Ref. [四], our results offer a tool for making a prediction of the proton decay rate with accuracy of two-loop level.

\section{Renormalization Factors}

In this section, we present the formula for the renormalization factors at the two loop level. They are derived from the effective Kähler potential given in Ref. [B]. In the calculation, the dimensional reduction scheme $(\overline{\mathrm{DR}})$ [捣] is employed for the regularization. The radiative corrections to the Kähler potential at two-loop level are described by

$$
\begin{aligned}
& \Delta K_{2}=\frac{1}{2} R_{a c}^{b d} J_{b d}^{a c}\left(M^{2}\right)-\sum_{\alpha} f_{A B C}^{(\alpha)} f_{D E F}^{(\alpha)} I^{B D E A F C}\left(M_{V(\alpha)}^{2}\right) \\
& -\sum_{\alpha}\left(G T_{A}^{(\alpha)} \phi\right)_{; c}^{b}\left(\bar{\phi} T_{B}^{(\alpha)} G\right)_{a}^{; d} H_{b d}^{a c} c^{A B}\left(M^{2}, M_{V(\alpha)}^{2}\right),
\end{aligned}
$$

with $f_{A B C}^{(\alpha)}$ the structure constants of the gauge group $\alpha$. The mass functions and the geometric factors appear in Eq. ([2]) are displayed in Ref.[[]]. By using them, we readily obtain the two-loop corrections to the vertex functions.

The RGE at two-loop level is given as

$$
\mu \frac{\partial \Gamma_{\mathscr{O}}^{(2)}}{\partial \mu}+\sum_{\alpha} \frac{1}{16 \pi^{2}} b_{\alpha} g_{\alpha}^{3} \frac{\partial}{\partial g_{\alpha}} \Gamma_{\mathscr{O}}^{(1)}-\sum_{i} \gamma_{i}^{(1)} \Gamma_{\mathscr{O}}^{(1)}-\sum_{i} \gamma_{i}^{(2)} \Gamma_{\mathscr{O}}^{(0)}+\gamma_{\mathscr{O}}^{(1)} \Gamma_{\mathscr{O}}^{(1)}+\gamma_{\mathscr{O}}^{(2)} \Gamma_{\mathscr{O}}^{(0)}=0 .
$$

Here, the subscripts (0-2) indicate the quantities are evaluated at tree, one-loop, and two-loop level, respectively. One-loop anomalous dimensions $\gamma_{i}^{(1)}$ are given as

$$
\gamma_{i}^{(1)}=-2 \sum_{\alpha} C_{\alpha}(i) \frac{g_{\alpha}^{2}}{16 \pi^{2}}
$$

while the two-loop ones are given as [囫]

$$
\gamma_{i}^{(2)}=\frac{1}{\left(16 \pi^{2}\right)^{2}} \sum_{\alpha, \beta} 2 g_{\alpha}^{2} C_{\alpha}(i)\left[g_{\alpha}^{2} b_{\alpha} \delta_{\alpha \beta}+2 g_{\beta}^{2} C_{\beta}(i)\right] .
$$


Here, $b_{\alpha}$ are the one-loop beta function coefficients for gauge coupling constants, given as $b_{\alpha}=$ $\sum_{i} I_{\alpha}(i)-3 C_{\alpha}(G)$ with $C_{\alpha}(G)$ and $I_{\alpha}(i)$ the quadratic Casimir invariant for the adjoint representation of the group $\alpha$ and the Dynkin index of the chiral multiplet $\Phi_{i}$, respectively.

From the RGE in Eq. (․2]), we now obtain the two-loop anomalous dimensions for the effective operators. Again, we parametrize them as follows:

$$
\begin{aligned}
\gamma_{\mathscr{O}^{(I)}}^{(2)} & =\frac{g_{3}^{4}}{\left(16 \pi^{2}\right)^{2}}\left[\gamma_{\mathscr{O}^{(I)}}^{(2)}\right]_{33}+\frac{g_{2}^{4}}{\left(16 \pi^{2}\right)^{2}}\left[\gamma_{\mathscr{O}^{(I)}}^{(2)}\right]_{22}+\frac{g_{Y}^{4}}{\left(16 \pi^{2}\right)^{2}}\left[\gamma_{\mathscr{O}^{(I)}}^{(2)}\right]_{Y Y} \\
& +\frac{g_{2}^{2} g_{3}^{2}}{\left(16 \pi^{2}\right)^{2}}\left[\gamma_{\mathscr{O}^{(I)}}^{(2)}\right]_{23}+\frac{g_{Y}^{2} g_{2}^{2}}{\left(16 \pi^{2}\right)^{2}}\left[\gamma_{\mathscr{O}^{(I)}}^{(2)}\right]_{Y 2}+\frac{g_{Y}^{2} g_{3}^{2}}{\left(16 \pi^{2}\right)^{2}}\left[\gamma_{\mathscr{O}^{(I)}}^{(2)}\right]_{Y 3}
\end{aligned}
$$

Then, we have

$$
\begin{array}{ll}
{\left[\gamma_{\mathscr{O}^{(1)}}^{(2)}\right]_{33}=\left[\gamma_{\mathscr{O}^{(2)}}^{(2)}\right]_{33}=\frac{64}{3}+8 b_{3},} & {\left[\gamma_{\mathscr{O}^{(1)}}^{(2)}\right]_{22}=\left[\gamma_{\mathscr{O}^{(2)}}^{(2)}\right]_{22}=\frac{9}{2}+3 b_{2},} \\
{\left[\gamma_{\mathscr{O}^{(1)}}^{(2)}\right]_{Y Y}=\frac{113}{54}+\frac{5}{3} b_{Y},} & {\left[\gamma_{\mathscr{O}^{(2)}}^{(2)}\right]_{Y Y}=\frac{91}{18}+3 b_{Y},} \\
{\left[\gamma_{\mathscr{O}^{(1)}}^{(2)}\right]_{23}=12,} & {\left[\gamma_{\mathscr{O}^{(2)}}^{(2)}\right]_{23}=20} \\
{\left[\gamma_{\mathscr{O}^{(1)}}^{(2)}\right]_{Y 2}=2,} & {\left[\gamma_{\mathscr{O}^{(2)}}^{(2)}\right]_{Y 2}=\frac{2}{3}} \\
{\left[\gamma_{\mathscr{O}^{(1)}}^{(2)}\right]_{Y 3}=\frac{68}{9},} & {\left[\gamma_{\mathscr{O}^{(2)}}^{(2)}\right]_{Y 3}=\frac{76}{9} .}
\end{array}
$$

\section{Results}

In this section, we give the numerical results of the renormalization factors in the minimal SUSY SU(5) GUT. The short-distance renormalization factors $A_{S}^{(I)}$ are defined as the ratios of the coefficients $C^{(I)}$ for the effective operators at the SUSY scale $M_{\mathrm{SUSY}}$ to those at the GUT scale $M_{\mathrm{GUT}}$ :

$$
A_{S}^{(I)} \equiv \frac{C^{(I)}\left(M_{\mathrm{SUSY}}\right)}{C^{(I)}\left(M_{\mathrm{GUT}}\right)}, \quad(I=1,2)
$$

where we assume $M_{\mathrm{SUSY}}=1 \mathrm{TeV}$ and $M_{\mathrm{GUT}}=1.5 \times 10^{16} \mathrm{GeV}$. The numerical results at one-loop level are given as

$$
\begin{aligned}
& A_{S}^{(1)}(1 \text {-loop })=1.959, \\
& A_{S}^{(2)}(1 \text { loop })=2.058,
\end{aligned}
$$

while at two-loop level, we have found

$$
\begin{aligned}
& A_{S}^{(1)}(2 \text {-loop })=1.961, \\
& A_{S}^{(2)}(2 \text {-loop })=2.052 .
\end{aligned}
$$

Here, we calculate the one-loop (two-loop) short-distance factors with the one-loop (two-loop) renormalization equations for the gauge coupling constants in the SUSY SM[目]. The numerical values of the unified gauge coupling constant at the one- and two-loop level are given as $\alpha_{5}$ (1-loop) $=$ 0.03906 and $\alpha_{5}(2$-loop $)=0.03968$, respectively, where $\alpha_{5}$ is defined as $\alpha_{5} \equiv g_{3}^{2}\left(M_{\mathrm{GUT}}\right) / 4 \pi$. The 
results are hardly affected by the uncertainty of the input parameters, e.g., the SU(3) gauge coupling constant, $\alpha_{s}\left(m_{Z}\right)=0.1184(7)[\square]$. There is a cancellation among the two-loop corrections since the signs of $\left[\gamma_{\mathscr{O}^{(1)}}^{(2)}\right]_{33}$ and $\left[\gamma_{\mathscr{O}^{(2)}}^{(2)}\right]_{33}$ are opposite to those of the other two-loop anomalous dimensions. Therefore, the numerical values at two-loop level hardly differ from the one-loop ones. Without cancellations, the significance of the two-loop contributions to the short-distance factors reaches a few percent of the one-loop ones.

\section{Conclusion and discussion}

We have evaluated the short-distance renormalization factors for the dimension-six proton decay operators at two-loop level with the effective Kähler potential. The procedure described in this Letter is generic and applicable to any higher-dimensional operators. We get the results $A_{S}^{(1)}(2$-loop $)=1.961$ and $A_{S}^{(2)}(2$-loop $)=2.052$ in the minimal SUSY SU(5) GUT. We have found that the two-loop contributions hardly change the renormalization factors evaluated at one-loop level.

Finally, we briefly comment on the extensions of the minimal SUSY GUT. The gauge coupling constants at the GUT scale increase if there exist extra particles in the intermediate scale. The twoloop effects may be more significant in such cases. In addition, let us note that our results are only for the $\mathrm{SU}(3)_{C} \times \mathrm{SU}(2)_{L} \times \mathrm{U}(1)_{Y}$ gauge interactions. If some new gauge interactions exist below the GUT scale, we also need to evaluate the contributions of the gauge interactions. Even for such theories, however, it is possible to execute the prescription describe above to estimate the renormalization factors by means of the effective Kähler potential.

In this Letter, we neglect the possible effects of the threshold corrections from particles whose masses are around the GUT scale. Although the effects are model-dependent, to complete the two-loop level calculation, we also need to evaluate such corrections.

\section{References}

[1] T. Nihei and J. Arafune, Prog. Theor. Phys. 93, 665 (1995) .

[2] C. Munoz, Phys. Lett. B 177, 55 (1986).

[3] S. Nibbelink Groot and T. S. Nyawelo, JHEP 0601, 034 (2006).

[4] W. Siegel, Phys. Lett. B 84, 193 (1979).

[5] J. Hisano, D. Kobayashi, Y. Muramatsu and N. Nagata, Phys. Lett. B 724, 283 (2013) [arXiv:1302.2194 [hep-ph]].

[6] S. P. Martin and M. T. Vaughn, Phys. Rev. D 50, 2282 (1994) [Erratum-ibid. D 78, 039903 (2008)].

[7] J. Beringer et al. [Particle Data Group Collaboration], Phys. Rev. D 86, 010001 (2012). 